\title{
SINGULAR INTEGRALS ON HILBERT SPACE
}

\author{
BY MICHAEL J. FISHER ${ }^{1}$
}

Communicated by Mark Kac, January 4, 1967

Introduction. Let $H$ be a real separable Hilbert space and let $1<p<\infty$. Let $y \rightarrow T_{y}$ denote the strongly continuous representation of the additive group of $H$ as a group of isometries on $L^{p}(H$, normal distribution) defined by $\left(T_{y} f\right)(x)=f(x-y) D(x, y)$ when $f$ is a bounded tame function on $H$ and

$$
D(x, y)=\exp \left[\frac{(x, y)}{p}-\frac{\|y\|^{2}}{2 p}\right] .
$$

If $\mu$ is a Borel measure on $H$ of bounded variation, let $\mu_{p}$ denote the strong integral $\int_{H} T_{y} d \mu(y)$. It is the object of this paper to give sufficient conditions on a complex measure $\mu$ of bounded variation on $H$ such that if $0<\delta<\rho<\infty$ and if

$$
Z^{\delta \rho}(E)=\int_{\delta}^{\rho} \mu(E / t) d t / t
$$

then the strong limit of the

$$
Z_{p}^{\delta \rho}=\int_{H} T_{y} d Z^{\delta \rho}(y)
$$

exists as a bounded operator on $L^{p}(H)$ as $\delta$ tends to zero and $\rho$ tends to infinity.

A theorem of this type extends the Calderon-Zygmund theory of singular integral operators on $E_{n}$ to infinite dimensions. For if $k(x)\|x\|^{-n}$ is a Calderon-Zygmund kernel and if $E$ is a bounded Borel set which is disjoint from a neighborhood of the origin then $\nu(E)$ $=\int_{E} k(x)\|x\| \|^{-n} d x$ satisfies $\nu(t E)=\nu(E)$ for $t>0$; if $g(x)$ is an in tegrable radial function on $E_{n}$ with support in a bounded annulus disjoint from a neighborhood of the origin, then $\int_{E_{n}} g(x) k(x)\|x\|-n d x=0$. When $\mu$ satisfies a smoothness condition and $\mu(H)=0$, the set function $\nu(E)=\int_{0}^{\infty} \mu(E / t) d t / t$ has these properties.

The results in this paper are taken from the author's Cornell doctoral dissertation. The author wishes to express his most hearty thanks to his thesis advisor Professor Leonard Gross for his interest, advice, and encouragement during the preparation of the thesis.

1 This research was supported in part by the National Science Foundation. 


\section{The main results.}

Definition 1. Let $\mu$ be a complex Borel measure of bounded variation on $H$, let $Z^{\delta \rho}$ be as in Equation (1) and $Z_{p}^{\delta \rho}$ be as in Equation (2) for $0<\delta<\rho<\infty$. The strong limit, $Z_{p}$, of the $Z_{p}^{\delta \rho}$ as $\delta \rightarrow 0$ and $\rho \rightarrow \infty$, if it exists, is the singular integral operator determined by $\mu$.

Let $n$ denote the normal distribution on $H$ and let $B$ be a one-one Hilbert-Schmidt operator on $H$. Then $n \circ B^{-1}$ is a Borel probability measure on $H$ (see [4, Corollary 3.2, p. 24]). In what follows we shall consider measures $d \mu(x)=a(x) d n \circ B^{-1}(x)$ where $a(x)$ is absolutely in tegrable with respect to $n \circ B^{-1}$.

TheOREM 1. Let $d \mu(x)=a(x) d n \circ B^{-1}(x)$ and $Z^{\text {op }}$ be as in Equation (1) and consider the operators $Z_{2}^{\delta \rho} f=\int_{H} T_{y} f d Z^{\delta \rho}(y)$ on $L^{2}(H)$. If $a \in \log ^{+} L\left(H, n \circ B^{-1}\right)$ and $\int_{H} a(x) d n \circ B^{-1}(x)=0$, then the strong limit, $Z_{2}$, of the $Z_{2}^{\delta \rho}$ exists as $\delta \rightarrow 0$ and $\rho \rightarrow \infty$ and

$$
\left\|Z_{2}\right\| \leqq K_{1} \int_{H}|a(x)| \log ^{+}|a(x)| d n \circ B^{-1}(x)+K_{2}
$$

where $K_{1}$ and $K_{2}$ are finite constants which do not depend on $a(x)$. If in addition, $a \in L^{r}\left(H, n \circ B^{-1}\right)$ for some $r>1$, then $\left\|Z_{2}\right\| \leqq D_{r}\|a\|_{r}$ where $D_{r}$ is a finite constant which depends only on $r$ and $\|a\|_{r}$ is the norm of $a(x)$ in $L^{r}\left(H, n \circ B^{-1}\right)$.

Method of Proof. Denote by $W$ the Wiener transform (see [5, pp. 119-123]) on $L^{2}(H)$. Then $W\left(Z_{2}^{\delta \rho} f\right)(\cdot)=\hat{Z}^{\delta \rho}\left(\frac{1}{2} \cdot\right)^{\sim} W(f)(\cdot)$ where $\hat{Z}^{\delta \rho}(\cdot) \sim$ is the measurable function on $H$ corresponding to the Fourier transform $\hat{Z}^{\delta \rho}(y)$ of the measure $Z^{\delta \rho}$. The $Z_{2}^{\delta \rho}$ converge strongly on $L^{2}(H)$ if and only if the $\hat{Z}^{\delta \rho}\left(\frac{1}{2} \cdot\right)^{\sim}$ converge boundedly and in measure with respect to the normal distribution on $H$. The desired conclusions now follow by direct computation.

Let $\mathfrak{F}$ denote the directed set of finite dimensional projections on $H$ and let $\mu$ be as in Theorem 1 .

Definition 2. For $Q$ in $\mathcal{F}$, the tame singular integral operator, $\left(Z \circ Q^{-1}\right)_{p}$, determined by $\mu$ is the strong limit, if it exists, of the tame integral operators $\left(Z \circ Q^{-1}\right)_{p}^{\delta \rho} f=\int_{H} T_{y} f d Z^{\delta \rho} \circ Q^{-1}(y)$ as $\delta$ tends to zero and $\rho$ tends to infinity, where $Z^{\delta \rho}$ is as in Equation (1).

Under the hypotheses of Theorem 1, the tame singular integral operators $\left(Z \circ Q^{-1}\right)_{2}$ exists and are uniformly bounded in $Q$.

Theorem 2. Let $d \mu(x)=a(x) d n \circ B^{-1}(x)$, suppose $\mu(H)=0$, and let $a \in L^{r}\left(H, n \circ B^{-1}\right)$ for some $r>1$. Let $Z_{2}$ be the singular integral operator determined by $\mu$ as in Theorem 1 and let $\left\{\left(Z \circ Q^{-1}\right)_{2} \mid Q \in \mathfrak{F}\right\}$ be the net of tame singular integral operators determined by $\mu$. Then this net converges strongly to $Z_{2}$ as $Q$ tends strongly to the identity through $\Im$. 
For the reflexive $L^{p}$-spaces there is

Theorem 3. Let $a(x), B, \mu, Z^{\delta \rho}$, and $Z_{p}^{\delta \rho}$ be as above. Then if $a \in L^{1}\left(H, n \circ B^{-1}\right)$ is an odd function, the strong limit, $Z_{p}$, of the $Z_{p}^{\text {op }}$ exists as $\delta \rightarrow 0$ and $\rho \rightarrow \infty$ and $\left\|Z_{p}\right\| \leqq G_{p}\|a\|_{1}$ where $G_{p}$ is a finite constant which depends only on $p$. If $a(x)$ is an even tame function in $L^{r}\left(H, n \circ B^{-1}\right)$ for some $r>1$ such that $\int_{H} a(x) d n \circ B^{-1}(x)=0$, then the strong limit, $Z_{p}$, of the $Z_{p}^{\delta \rho}$ exists as $\delta \rightarrow 0$ and $\rho \rightarrow \infty$ and $\left\|Z_{p}\right\|$ $\leqq K(r, p)\|a\|_{r}$ where $K(r, p)$ is a finite constant which depends on $r, p$, and the dimension of the base space of $a(x)$.

REMARK. We have not stated Theorem 3 in the most general form in which we know it to hold since we do not want to introduce new complicated notation in this paper. If $\mu$ is an odd Borel measure of bounded variation on $H$, then $\mu$ determines a bounded singular integral operator, $Z_{p}$, as above and $\left\|Z_{p}\right\| \leqq G_{p}\|\mu\|$. In the case in which the function $a(x)$ is even, greater generality is achieved by writing $a(x)$ as a series, $a(x)=\Sigma_{i} a_{i}(x)$, where the vector-valued integral of $a_{i}(x)$ over a certain finite dimensional subspace of $H$ (depending on $i$ ) vanishes. The set of functions $a(x)$ for which this series converges absolutely in $L^{r}\left(H, n \circ B^{-1}\right)$ forms a Banach space $N^{r}\left(H, n \circ B^{-1}\right)$ $C L^{r}\left(H, n \circ B^{-1}\right)$ which contains nontame functions and is such that if $a \in N^{r}\left(H, n \circ B^{-1}\right)$ then $a(x)$ determines a bounded singular integral operator, $Z_{p}$, as above with $\left\|Z_{p}\right\| \leqq K N^{r}(a)$ where $N^{r}(a)$ is the norm in $N^{r}\left(H, n \circ B^{-1}\right)$ and $K$ is a finite constant which depends only on $r$ and $p$.

Method of Proof of Theorem 3. When $a(x)$ is an odd function, we apply Minkowski's integral inequality, M. Riesz' theorem on the Hilbert transform, and the dominated convergence theorem.

When $a(x)$ is an even function, a special argument is needed. Let $F$ denote the base of $a(x)$ and $G$ denote the image of $F$ under $B$. Let $P_{0}$ denote the orthogonal projection from $H$ to $G$. Let $\odot=\left\{P_{n}\right\}$ be an ordered sequence $P_{0}<P_{1}<P_{2}<\cdots$ of finite dimensional orthogonal projections which converge strongly to the identity. Consider the tame operators $\left(Z \circ Q^{-1}\right)_{p}^{\delta \rho}$ determined by $a(x)$ and $Q \in \odot, Q>P_{0}$. We compose $\left(Z \circ Q^{-1}\right)_{p}^{\delta \rho}$ with certain Calderon-Zygmund operators $\left\{R_{p}^{k}\right\}$ on $L^{p}(H)$ which have odd kernels and which have the properties that $\sum_{k=1}^{N} R_{p}^{k}\left(R_{p}^{k}\left(Z \circ Q^{-1}\right)_{p}^{\delta \rho} f\right)=-\left(Z \circ Q^{-1}\right)_{p}^{\delta \rho} f$, where $N$ is the dimension of $F$, and $R_{p}^{k}\left(Z \circ Q^{-1}\right)_{p}^{\delta \rho} f=\left(H \circ Q^{-1}\right)_{p k}^{\delta \rho} f$ where $\left(H \circ Q^{-1}\right)_{p k}^{\delta \rho} f$ is an approximate tame singular integral operator determined by an odd $L^{1}$-function. By the dominated convergence theorem, $\left(Z \circ Q^{-1}\right)_{p}^{\delta \rho}$ and $\left(H \circ Q^{-1}\right)_{p k}^{\delta \rho}$ converge strongly to $Z_{p}^{\delta \rho}$ and $H_{p k}^{\delta \rho}$, respectively, as $Q$ tends strongly to the identity through $P$, where $H_{p k}^{\delta \rho}$ is an approximate 
singular integral operator determined by an odd $L^{1}$-function. The result now follows from the case when $a(x)$ is odd and a special estimate for the $L^{1}$-norm of the function determining $H_{p k \text {. }}^{\delta \rho}$.

Suppose that the Hilbert space, $H$, is $N$-dimensional Euclidean space, $E_{N}$.

THEOREM 4. Let $a \in L^{r}\left(E_{N}, n \circ B^{-1}\right)$ for some $r>1$ and $\int_{E_{N}} a(x) d n \circ B^{-1}(x)=0$. Then there is a finite complex constant

$$
A=\int_{E_{N}}-\log \|x\| a(x) d n \circ B^{-1}(x)
$$

and a unique Calderon-Zygmund operator $C_{p}$ (appropriately transposed to $L^{p}\left(E_{N}, n\right)$ ) such that $A f+C_{p} f=Z_{p} f$ where $Z_{p}$ is the singular integral operator of Theorem 3 determined by $a(x) . C_{p}$ has kernel $S(a)(y)\|y\|^{-N}$ where

$S(a)(\omega)=\left((2 \pi)^{N / 2} \operatorname{det} B\left\|B \omega^{-1}\right\|^{N}\right)^{-1}\left(\int_{0}^{\infty} \Omega\left(s B \omega^{-1}\left\|B \omega^{-1}\right\|^{-1}\right) \exp \frac{-s^{2}}{2} s^{N-1} d s\right.$ for $\|\omega\|=1$ and $\Omega(x)=a(B x)$. Furthermore,

$$
\|S(a)\|_{L^{r(\Sigma, \sigma)}} \leqq K(r, N)\|a\|_{L^{r}\left(E_{N}, n^{, B^{-1}}\right)}
$$

where $\sigma$ denotes Lebesgue measure on the unit sphere, $\Sigma$, and $K(r, N)$ is a finite constant depending only on $r$ and $N$.

This theorem is proved by direct computation.

REMARK. It follows from Theorem 4 that if the operator $B$ on $E_{N}$ is the identity operator and if $a(x)$ is homogeneous of degree zero, then the singular integral operator $Z_{p}$ determined by $a(x)$ is the CalderonZygmund operator $C_{p}$ and $C_{p}$ has kernel const. $a(y)\|y\|-N$. Furthermore, every Calderon-Zygmund operator arises in this way.

\section{REFERENCES}

1. A. Calderon and A. Zygmund, Algebras of certain singular operators, AmerJ. Math. 78 (1956), 310-320.

2. M. J. Fisher, Integration and nonlinear transformations in Hilbert space, $\mathrm{Ph} . \mathrm{D}$. Thesis, Cornell University, Ithaca, New York, 1966.

3. L. Gross, Integration and nonlinear transformations in Hilbert space, Trans. Amer. Math. Soc. 94 (1960), 404-440.

4. — Harmonic analysis on Hilbert space, Mem. Amer. Math. Soc. No. 46 (1963).

5. I. E. Segal, Tensor algebras over Hilbert spaces, Trans. Amer. Math. Soc. 81 (1956), 106-134.

6. - Distributions in Hilbert space and canonical systems of operators, Trans. Amer. Math. Soc. 88 (1958), 12-41.

The Johns Hopkins University 